\title{
Single Crystal Aluminum Deposit Formation on Isotropic Substrates by Means of Self-Organized Ion Sputtering
}

\author{
V. I. Perekrestov*, A. S. Kornyushchenko, and Yu. A. Kosminskaya \\ Sumy State University, Sumy, 40007 Ukraine \\ e-mail: kpe@sumdu.edu.ua \\ Received April 4, 2006
}

\begin{abstract}
A substantially new technological approach is considered, which is based on the self-organized formation of aluminum single crystal layer on isotropic substrates in the course of stationary condensation of reverse diffusion fluxes in a planar dc magnetron. The functional possibilities of self-organized ion sputtering systems are discussed.
\end{abstract}

PACS numbers: 81.10.-h

DOI: $10.1134 / \mathrm{S} 1063785006100154$

Proceeding from the of small model nuclei originally proposed by Walton and Rhodin [1,2], it is possible to establish a qualitative relationship between the number of atoms in a critical nucleus and the chemical potential difference $\Delta \mu$ between the gas (vapor) and condensed phases [3]. This relationship shows that, in the general case, there are three variants of condensation from the vapor phase. First, in the region of high supersaturation (large $\Delta \mu$ ), the condensation proceeds via statistical deposition of single-atom critical nuclei. As the degree of supersaturation decreases, the deposition proceeds by the second variant, whereby polyatomic critical nuclei are formed in which the number of atoms is proportional to $1 / \Delta \mu$ [4]. The third, most important variant is realized in the case of ultimately small supersaturations (and $\Delta \mu$ values). Under such conditions, the diffusion field of adatoms is weak so that the formation of polyatomic critical nuclei on the growth surface is practically excluded. In this case, the growth proceeds via the attachment of single atoms at the active centers on the growth surface, where the binding energy exceeds a certain critical value $\left(E_{\mathrm{s}}\right)$ [3].

The third variant of condensation is realized in the technology of layer growth by means of molecular beam epitaxy (MBE) [5], metalorganic chemical vapor deposition [6], crystallization from supersaturated solutions [7], and electrolytic deposition [8]. In particular, the proximity to phase equilibrium in the vapor-condensate system in the course of MBE is achieved by deposition from weak flows at high temperatures. In other cases [6-8], these conditions are provided by the chemical interaction of adatoms with the environment, which favors their reverse transition to the solution or the gas phase. Such processes are less sensitive to temperature variations, which is evidence for a kind of self- organization. In this context, a promising approach consists in the creation of analogous, more universal self-organized systems based on physical principles. This Letter considers a possible realization of this approach.

Self-organized ion sputtering. The process of condensation by means of Self-organized ion sputtering is based on the energy transfer from a flux of particles bombarding the growth surface to adatoms, which increases the probability of their reverse passage to the gas phase $[3,9]$. The self-regulation of the technological parameters of condensation ensures the proximity to phase equilibrium in the environment-condensate system and determines a stationary character of the deposition process. The energy of particles acting upon the growth surface must not exceed a threshold energy for the sputtering of a condensed crystalline deposit.

We have developed a variant of the self-organized ion sputtering system based on a planar dc magnetron. In the proposed setup (Fig. 1a), target 3 is sputtered in the region of crossed electric and magnetic fields created by electrodes and permanent magnets 1 . Prior to deposition, the working chamber was outgassed and the working gas (argon) was thoroughly purified so that the partial pressure of chemically active residual gases was reduced below $10^{-8} \mathrm{~Pa}$ as described in [10]. Then, drive 6 is moved down to open shutter 5 and allow the sputtered substance to deposit by reverse diffusion onto substrate 4 , which is fixed at the center of water-cooled table 2 .

The required conditions of reverse diffusion can be provided at an argon pressure above 5-7 $\mathrm{Pa}$, which also decreases and levels the energies of deposited atoms and argon ions [11]. The self-organized proximity to phase equilibrium was achieved through synchronous 
(a)

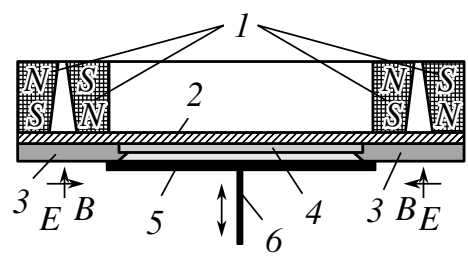

(b)

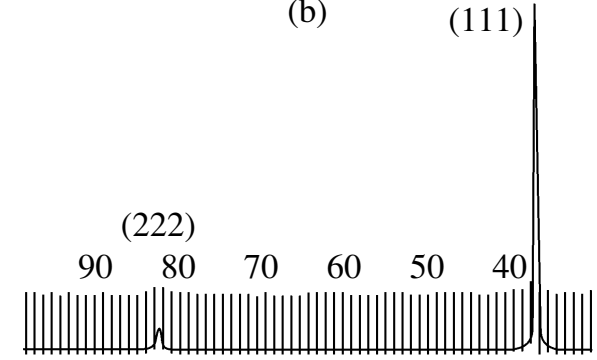

Fig. 1. Self-organized ion sputtering system: (a) schematic diagram of the experimental setup (see text for explanations); (b) the typical X-ray diffractogram of an aluminum layer grown in this system.
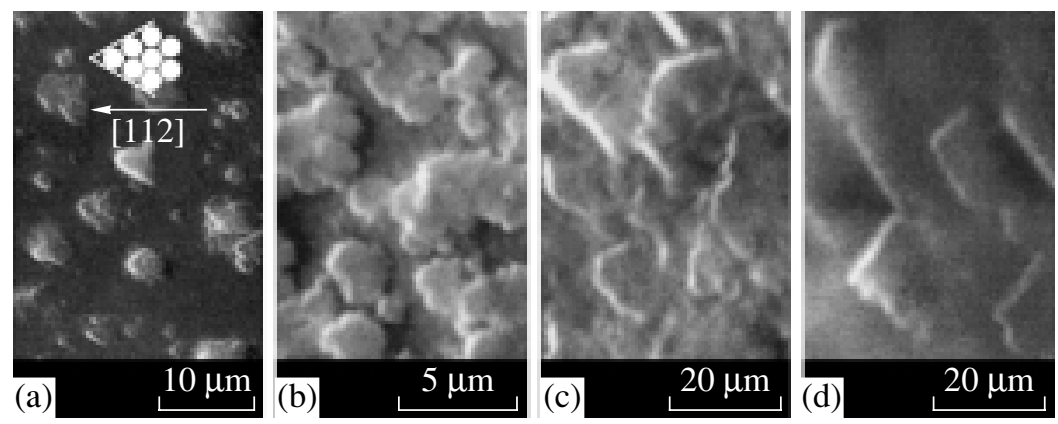

Fig. 2. Scanning electron microscope images illustrating various stages of aluminum layer formation in the course of self-organized ion sputtering, which correspond to the deposition time of (a) 0.5 , (b) 1.2 , (c) 2 , and (d) $10 \mathrm{~h}$.

variation of the main technological parameters, including the growth surface temperature, deposited material flux density, and the energy and density of particles bombarding the growth surface. In particular, a change (within definite limits) in the discharge power leads to simultaneous consistent variation in the deposited flux and ion plasma density, which provides to a certain extent the constancy of $E_{\mathrm{s}}$. It should be emphasized that re-evaporated or re-sputtered atoms are most probably ionized and re-deposited, and this mass transfer cycle can be multiply repeated until the binding energy will exceed $E_{\mathrm{s}}$. Each operating mass-transfer circle is a "diligent creator" of the desired structure. In this context, the atom-by-atom building of the deposit controlled by the electric field and the $E_{\mathrm{s}}$ value can be considered as a nanotechnology process.

Let us consider the sequential stages of formation of a single crystal layer of aluminum on a glass substrate at a discharge power of $\sim 40 \mathrm{~W}$ and an argon pressure of $15 \mathrm{~Pa}$. Thorough analysis of the stage of deposit nucleation (Fig. 2a) shows that the nuclei have approximately the same [112] orientation in the substrate plane. This predominant orientation is confirmed by the presence of $60^{\circ}$ corners aligned in approximately the same direction (Fig. 2a) and is probably related to the existence of a tangential component of the electric field over the dielectric substrate surface. It should be noted that, on the whole, the same orientation of growing crystals is possible only provided that at least one addi- tional preferred crystallographic direction exists at the substrate plane. Indeed, the X-ray diffraction data showed that the crystal growth normal to the substrate surface proceeds only in the [111] direction (Fig. 1b), which can be explained in terms of minimization of the free energy at the condensate-substrate interface. It should be emphasized that the same orientation of growing crystals relative to the isotropic substrate is possible only under the conditions of nucleation at the active centers and in the form of single-atom critical nuclei, since a control over the orientation of polyatomic critical nuclei is hardly possible. Using the known method of deposited flux calculation [11] and evaluating the deposited material volume from microscopic data (Fig. 2a), it is possible to show that, even by overstated estimates, only about $1-7 \%$ of the deposited $\mathrm{Al}$ atoms are condensed upon the first trial. This fact is another evidence for the proximity to phase equilibrium in the vapor-condensate system under consideration.

The subsequent intergrowth of the nuclei (Figs. 2b-2d) leads to the formation of layer with an almost single crystal structure, which is confirmed by the electronmicroscopic images (showing the presence of a considerable number of identically oriented growth steps on the growth surface) and by the X-ray diffraction patterns. In view of the isotropic character of the substrate surface, a network of misfit dislocations is apparently formed when the separate crystals grow together. In addition, predominant growth of particular steps (Fig. 2c) leads to their superimposition over adjacent 
separate crystals, which eventually favors the formation of a more perfect structure.

Functional possibilities of self-organized ion sputtering systems. Advantages of the self-organized ion sputtering process over the existing widely used methods of crystal layer growth [5-8] are as follows:

(i) Self-organized ion sputtering systems offer more possibilities in providing conditions both for the transfer of s substance to the gas phase and for the self-organized approach to phase equilibrium in the vapor-condensate system. For example, the equilibrium vapor pressure over aluminum at a melting temperature is as low as $10^{-7} \mathrm{~Pa}$ [12], which makes the MBE growth of this metal an extremely difficult task.

(ii) the presence of an electric field over the growth surface and the deposition of ionized species can favor the self-organized growth of not only single crystals, but of various columnar structures as well [13]. Thus, the electric field is an additional control parameter for the atomic assembly of condensates.

(iii) Under conditions of ultimately small condensation coefficients, the conditions of self-organized approach to phase equilibrium in the vapor-condensate system can be provided even for rather intense atomic flows. This makes possible the accelerated growth of layers with various architectural forms.

(iv) A decrease in the condensation coefficients is provided by direct energy transfer from ions bombarding the growth surface to adatoms, which allows the conditions close to phase equilibrium to be obtained on cooled substrate. Thus, it is possible to decrease the mutual diffusion of components in the course of heterojunction formation and to expand the nomenclature of substrates.

\section{REFERENCES}

1. T. Rhodin and D. Walton, in Single Crystal Films, Ed. By M. H. Francombe and H. Sato (Pargamon, London 1964), pp. 31-41.

2. D. Walton, T. Rhodin, and R. Rollings, J. Chem. Phys. 38, 2698 (1963).

3. V. I. Perekrestov, Pis'ma Zh. Tekh. Fiz. 31 (19), 41 (2005) [Tech. Phys. Lett. 31, 830 (2005)].

4. A. A. Chernov, in Modern Crystallography, Vol. 3: Crystal Growth, Ed. by B. K. Vainshtein, A. A. Chernov, and L. A. Shuvalov (Nauka, Moscow, 1980; Springer, Berlin, 1984).

5. K M. Herman, Semiconductor Superlattices (AkademieVerlag, Berlin, 1986).

6. V. B. Ufimtsev and R. Kh. Akchurin, Physicochemical Grounds of Liquid Phase Epitaxy (Metallurgiya, Moscow, 1983) [in Russian].

7. V. M. Fedosyuk, M. U. Sheleg, and O. I. Kasyutich, Zarubezhn. Radioélektron., No. 5, 88 (1990).

8. L. Feldman and D. Mayer, Fundamentals of Surface and Thin Film Analysis (North-Holland, New York, 1986).

9. V. I. Perekrestov and Yu. A. Kosminskaya, Pis'ma Zh. Éksp. Teor. Fiz. 78, 258 (2003) [JETP Lett. 78, 223 (2003)].

10. V. I. Perekrestov and S. N. Kravchenko, Prib. Tekh. Éksp., No. 3, 123 (2002).

11. B. S. Danilin, Application of Low-Temperature Plasma for Thin Film Deposition (Énergoatomizdat, Moscow, 1989) [in Russian].

12. Handbook of Thin Film Technology, Ed. by L. I. Maissel and R. Glang (McGraw-Hill, New York, 1970; Sov. Radio, Moscow, 1977).

13. V. I. Perekrestov and Yu. A. Kosminskaya, Metallofiz. Noveǐsh. Tekhnol. 27, 265 (2005).

Translated by P. Pozdeev

Spell: ok 